Article

\title{
A New Control Chart for Monitoring the Process Mean Using Successive Sampling and Multiple Dependent State Repetitive Sampling
}

\author{
Mansour Sattam Aldosari ${ }^{1}$, Muhammad Aslam ${ }^{1, * \mathbb{D}}$, Chi-Hyuck Jun ${ }^{2}$ and Khushnoor Khan ${ }^{1}$ \\ 1 Department of Statistics, Faculty of Science, King Abdulaziz University, Jeddah 21551, Saudi Arabia; \\ msattam@hotmail.com (M.S.A.); khushnoorkhan64@gmail.com (K.K.) \\ 2 Department of Industrial and Management Engineering, Pohang University of Science and \\ Technology (POSTECH), Pohang 37673, Korea; chjun@postech.ac.kr \\ * Correspondence: aslam_ravian@hotmail.com
}

Received: 18 June 2018; Accepted: 25 July 2018; Published: 30 July 2018

\begin{abstract}
In this paper, a new control chart scheme has been developed for monitoring the production process mean using successive sampling over two occasions. The proposed chart reduces to three different existing control charts under different assumptions and is compared with these three existing control charts for monitoring the process average. It has been observed that the proposed control chart performs better than the other existing control charts in terms of average run length $(A R L)$. A simulation study using an artificial data set was included for demonstrating the process shift detection power of the proposed control chart.
\end{abstract}

Keywords: successive sampling; auxiliary information; control chart; average run length; process mean

\section{Introduction}

Control charts are being used extensively for monitoring the manufacturing processes to detect any unusual change in the quality characteristic of interest. Timely and speedily investigation of the process shift and the corrective actions, communicated by the control charts, are useful measures toward bringing the process back into statistical control. The control chart is an effective on-line monitoring scheme extensively used for this purpose [1,2]. Two types of control charts are most commonly used in the literature of the process control: one is used for monitoring the process mean or location (for example, $\bar{X}-$ Chart), and the other is used for monitoring the variability or dispersion (for example, $R$ - Chart) [3]. In the beginning, the design structure of these charts was based upon simple random sampling, but recently, many techniques have been proposed regarding the designs and sampling schemes.

Successive sampling has been used extensively in applied and social sciences for estimating the mean of the finite population and attracted the attention of many researchers during the last two decades, for more details, see [1,4-11]. The idea of successive sampling on two occasions was introduced by [12]. The use of successive sampling in the area of control charts increases its monitoring ability substantially (see for example [13]). The preparation of surveying the population for estimating the population parameter at different time points is called sampling over successive occasions [4]. In sampling over successive occasions on the matched portion of the sample, the information about the target/interested quality characteristics is collected from the sample of the current occasion, and the information from the preceding samples is used as the auxiliary information. The utilization of the auxiliary information for estimating the parameter using the current situation of population only on two successive occasions have been explored by many authors, including $[4,7,14]$. 
The performance evaluation of any proposed chart is examined by Average Run Length $(A R L)$, which may be defined as the average number of samples before the process indicates an out-of-control process [2]. The $A R L$ is an important tool of process design and performance evaluation [15]. There are several methods including the Markov Chain approach, integral equation approach, and Monte Carlo simulations to calculate the in-control $A R L\left(A R L_{0}\right)$ and $A R L$ of the out-of-control $\left(A R L_{1}\right)$ processes [16]. The value of $A R L_{0}$ is considered to be the higher, as the process is in a state of in-control, while a smaller value of $A R L_{1}$ is known to be better for the efficient monitoring of the process, as the out-of-control process is indicated quickly to avoid losses of scrap and/or rework. Several researchers used the $A R L$ calculation for examining the performance of the proposed scheme, including [17-20].

In this article, a control chart design has been proposed for monitoring the process mean using successive sampling over two occasions. In sampling over successive occasions on the matched portion of the sample, the information about the target/interested quality characteristics is collected from the sample of the current occasion, and the information from the preceding samples is used as the auxiliary information. The correlation coefficient here is the one between the main variable with an auxiliary one. One of the objectives in this study is to investigate the performance of the proposed chart according to the magnitude of this correlation coefficient. The rest of the article is organized as follows: The designing of the proposed chart is given in Section 2. In Section 3 the probability of in-control and out-of-control processes is described. The methodology of ARL of the in-control and the out-of-control is given in Section 4. In Section 5, a comparison of proposed chart with four existing charts has been discussed. Concluding remarks are given in the last Section.

\section{Designing of Proposed Control Chart}

Suppose that the main quality characteristic of interest is $Y$ with mean $\mu_{Y}$ and variance $\sigma_{Y}^{2}$, and that an auxiliary variable $X$ having mean $\mu_{X}$ and variance $\sigma_{X}^{2}$ is also measured from sampling. The correlation coefficient between $Y$ and $X$ is denoted by $\rho$. It is assumed that $\sigma_{X}^{2}=\sigma_{Y}^{2}=\sigma^{2}$ for simplicity. It is also to be noted that our main variable of interest to be monitored here is the $Y$ variable through discovered information of the $X$ variable. Here, we would like to improve the efficiency of the estimator of $\mu_{Y}$ using successive sampling over two occasions.

We draw two samples of size $n$ each at the first and the second occasions. Suppose that there are $m$ common (called matched) units from two occasions so that there are $u(n-m)$ unmatched units from the second occasion. Let $\bar{x}_{m}$ and $\bar{y}_{m}$ show the sample means of the matched units for $X$ and $Y$ variables, respectively. Let $\bar{x}_{u}$ and $\bar{y}_{u}$ be the sample means for the unmatched units for $X$ and $Y$ variables, respectively. Then, Mukhopadhyay proposed the following estimator of $\mu_{Y}$ :

$$
\hat{\mu_{Y}}=a \bar{x}_{u}+b \bar{x}_{m}+c \bar{y}_{m}+d \bar{y}_{u}
$$

where $a, b, c$, and $d$ are constant satisfying $a+b=0$ and $c+d=1$. A more details about the estimation of these constants can be seen in [11].

The variance of estimator under the assumption that population variances are equal is given by

$$
\operatorname{Var}\left(\hat{\mu_{Y}}\right)=\operatorname{Var}\left\{a\left(\bar{x}_{u}-\bar{x}_{m}\right)+c \bar{y}_{m}+(1-c) \bar{y}_{u}\right\}
$$

According to [21-23], the distribution of $\hat{\mu}_{Y}$ is given as

$$
\hat{\mu_{Y}} \sim N\left[\mu_{Y}, \frac{\sigma^{2}}{2 n}\left(1+\sqrt{1-\rho^{2}}\right)\right]
$$

The steps of the proposed control chart will be as follows:

Step 1

We draw two samples of size $\mathrm{n}$ each at the first and the second occasions. Suppose that there are $m$ common (called matched) units from two occasions so that there are $u(n-m)$ unmatched 
units from the second occasion. Let $\bar{x}_{m}$ and $\bar{y}_{m}$ be the sample means of the matched units for $X$ and $Y$ variables, respectively. Let $\bar{x}_{u}$ and $\bar{y}_{u}$ be the sample means for the unmatched units for $X$ and $Y$ variables, respectively.

Step 2

Compute the value of the estimator

$$
\hat{\mu_{Y}}=a \bar{x}_{u}+b \bar{x}_{m}+c \bar{y}_{m}+d \bar{y}_{u}
$$

(The algorithm of determining $a, b, c$ and $d$ is given in Section 3.)

Step 3 (Decision State):

Declare the process as in-control if $L C L_{2} \leq \hat{\mu_{Y}} \leq U C L_{2}$ and as out-of-control if $\hat{\mu_{Y}} \geq U C L_{1}$ or $\hat{\mu}_{Y} \leq L C L_{1}$. Otherwise, go to Step 4

Step 4 (Indecision State):

If $U C L_{2} \leq \hat{\mu_{Y}} \leq U C L_{1}$ or $L C L_{1} \leq \hat{\mu_{Y}} \leq L C L_{2}$. The process is declared as in-control if $i$ proceeding subgroups have been declared as in-control. Otherwise, repeat Step 1.

Where $L C L$ and $U C L$ show lower control limit and upper control limit, respectively.

The proposed control chart consists of four control limits according to the successive sampling estimator are namely $L C L_{1}, L C L_{2}, U C L_{1}, U C L_{2}$ having two control limits coefficients $k_{1}$ and $k_{2}$. The proposed control chart is an extension of several existing control charts. The proposed control chart reduces to the chart by [18] when $i=0$ and to the chart by [24] when $k_{1}=k_{2}$. The proposed chart becomes the chart by [25] when $i=0$ and $k_{1}=k_{2}$.

The two outer control limits for the proposed control chart are given as

$$
\begin{aligned}
& L C L_{1}=\mu_{0}-k_{1} \sqrt{\frac{\sigma^{2}}{2 n}\left(1+\sqrt{1-\rho^{2}}\right)} \\
& U C L_{1}=\mu_{0}+k_{1} \sqrt{\frac{\sigma^{2}}{2 n}\left(1+\sqrt{1-\rho^{2}}\right)}
\end{aligned}
$$

The two inner control limits are given as

$$
\begin{aligned}
& L C L_{2}=\mu_{0}-k_{2} \sqrt{\frac{\sigma^{2}}{2 n}\left(1+\sqrt{1-\rho^{2}}\right)} \\
& U C L_{2}=\mu_{0}+k_{2} \sqrt{\frac{\sigma^{2}}{2 n}\left(1+\sqrt{1-\rho^{2}}\right)}
\end{aligned}
$$

Here, $\mu_{0}$ is the population mean when the process is in control.

\section{Average Run Lengths}

The probability that the process is declared as in-control based on a single sample is given as follows:

$$
\begin{aligned}
P_{i n, 1}^{0}=P\left(L C L_{2}\right. & \left.\leq \hat{\mu_{Y}} \leq U C L_{2}\right) \\
& +\left\{P\left(L C L_{1}<\hat{\mu_{Y}}<L C L_{2}\right)\right. \\
& \left.+P\left(U C L_{2}<\hat{\mu}_{Y}<U C L_{1}\right)\right\}\left\{P\left(L C L_{2} \leq \hat{\mu_{Y}} \leq U C L_{2}\right)\right\}^{i}
\end{aligned}
$$

When the plotting statistic is in-decision state, the process is repeated as stated in Step 4 of proposed control chart. Let $P_{r e p}^{0}$ denote the probability for this area. Then, it is given as follows: 


$$
\begin{gathered}
P_{\text {rep }}^{0}=\left\{P\left(L C L_{1}<\hat{\mu}_{Y}<L C L_{2}\right)+P\left(U C L_{2}<\hat{\mu}_{Y}<U C L_{1}\right)\right\}(1 \\
\left.-\left[P\left\{L C L_{2} \leq \hat{\mu}_{Y} \leq U C L_{2}\right\}\right]^{i}\right)
\end{gathered}
$$

Let us define

$$
\begin{gathered}
A_{1}^{0}=P\left\{L C L_{2}<\hat{\mu_{Y}}\left\langle U C L_{2}\right| \mu_{Y}=\mu_{0}\right\}=2 \Phi\left(k_{2}\right)-1 \\
A_{2}^{0}=P\left(L C L_{1}<\hat{\varkappa}_{Y}\left\langle L C L_{2}\right| \mu_{Y}=\mu_{0}\right)=\Phi\left(k_{1}\right)-\Phi\left(k_{2}\right) \\
A_{3}^{0}=P\left(U C L_{2}<\hat{\Upsilon}_{Y}\left\langle U C L_{1}\right| \mu_{Y}=\mu_{0}\right)=\Phi\left(k_{1}\right)-\Phi\left(k_{2}\right)
\end{gathered}
$$

Then, the probability $P_{i n, 1}^{0}$ given in Equation (10) can be written as follows:

$$
P_{i n, 1}^{0}=A_{1}^{0}+\left\{A_{2}^{0}+A_{3}^{0}\right\}\left\{A_{1}^{0}\right\}^{i}
$$

or

$$
P_{i n, 1}^{0}=\left(2 \Phi\left(k_{2}\right)-1\right)+2\left\{\Phi\left(k_{1}\right)-\Phi\left(k_{2}\right)\right\}\left\{2 \Phi\left(k_{2}\right)-1\right\}^{i}
$$

The probability of repetition is given as

$$
P_{\text {rep }}^{0}=2\left\{\Phi\left(k_{1}\right)-\Phi\left(k_{2}\right)\right\}\left(1-\left[2 \Phi\left(k_{2}\right)-1\right]^{i}\right)
$$

or

$$
P_{\text {rep }}^{0}=\left\{A_{2}^{0}+A_{3}^{0}\right\}\left(1-\left[A_{1}^{0}\right]^{i}\right)
$$

The probability that the process is declared as in control for the proposed chart is given as follows

$$
P_{\text {in }}^{0}=\frac{P_{\text {in, } 1}^{0}}{1-P_{\text {rep }}^{0}}=\frac{A_{1}^{0}+\left\{A_{2}^{0}+A_{3}^{0}\right\}\left\{A_{1}^{0}\right\}^{i}}{1-\left\{A_{2}^{0}+A_{3}^{0}\right\}\left(1-\left[A_{1}^{0}\right]^{i}\right)}
$$

The average run length $(A R L)$ is one of the most useful performance measures for evaluating the efficiency of a control chart.

The ARL of the proposed control chart when the process is in control is defined as follows

$$
A R L_{0}=\frac{1}{1-P_{i n}^{0}}
$$

Now, we suppose that the process is shifted from $\mu_{0}$ to $\mu_{1}=\mu_{0}+\sigma f$; where ' $f$ ' indicates shift constant. Let $P_{i n, 1}^{1}$ denote the probability that the process is declared as in-control for the shifted process based on a single sample is given as

$$
\begin{aligned}
P_{i n, 1}^{1}=P\left(L C L_{2}\right. & \left.\leq \hat{\mu_{Y}} \leq U C L_{2} \mid \mu_{1}\right) \\
& +\left\{P\left(L C L_{1}<\hat{\mu}_{Y}\left\langle L C L_{2}\right| \mu_{1}\right)\right. \\
& +P\left(U C L_{2}<\hat{\mu_{Y}}\right. \\
& \left.\left.<U C L_{1} \mid \mu_{1}\right)\right\}\left\{P\left(L C L_{2} \leq \hat{\mu_{Y}} \leq U C L_{2} \mid \mu_{1}\right)\right\}^{i}
\end{aligned}
$$

The probability of repeated sampling, say $P_{r e p}^{1}$ at $\mu_{1}$ is given as

$$
\begin{gathered}
P_{\text {rep }}^{1}=\left\{P\left(L C L_{1}<\hat{\mu}_{Y}\left\langle L C L_{2}\right| \mu_{1}\right)+P\left(U C L_{2}<\hat{\mu}_{Y}\left\langle U C L_{1}\right| \mu_{1}\right)\right\}(1 \\
\left.\left.-\left[P\left\{L C L_{2} \leq \hat{\mu}_{Y} \leq U C L_{2} \mid \mu_{1}\right\}\right]\right]^{i}\right)
\end{gathered}
$$

Let us define

$$
\begin{aligned}
A_{1}^{1}=P\left(L C L_{2}\right. & \left.\leq \hat{\mu}_{Y} \leq U C L_{2} \mid \mu_{1}\right) \\
& =\Phi\left(k_{2}-\frac{f}{\sqrt{\frac{1}{2 n}\left(1+\sqrt{1-\rho^{2}}\right)}}\right) \\
& +\Phi\left(k_{2}+\frac{f}{\sqrt{\frac{1}{2 n}\left(1+\sqrt{1-\rho^{2}}\right)}}\right)-1
\end{aligned}
$$




$$
\begin{aligned}
A_{2}^{1}=P\left(L C L_{1}\right. & \left.<\hat{\mu}_{Y}\left\langle L C L_{2}\right| \mu_{1}\right) \\
& =\Phi\left(k_{1}+\frac{f}{\sqrt{\frac{1}{2 n}\left(1+\sqrt{1-\rho^{2}}\right)}}\right) \\
& -\Phi\left(k_{2}+\frac{f}{\sqrt{\frac{1}{2 n}\left(1+\sqrt{1-\rho^{2}}\right)}}\right) \\
A_{3}^{1}=P\left(U C L_{2}\right. & \left.<\hat{\mu}_{Y}\left\langle U C L_{1}\right| \mu_{1}\right) \\
& =\Phi\left(k_{1}+\frac{f}{\sqrt{\frac{1}{2 n}\left(1+\sqrt{1-\rho^{2}}\right)}}\right) \\
& -\Phi\left(k_{2}+\frac{f}{\sqrt{\frac{1}{2 n}\left(1+\sqrt{1-\rho^{2}}\right)}}\right)
\end{aligned}
$$

Then, Equation (22) can be written as

$$
P_{i n, 1}^{1}=A_{1}^{1}+\left\{A_{2}^{1}+A_{3}^{1}\right\}\left\{A_{1}^{1}\right\}^{i}
$$

or

$$
\begin{aligned}
P_{\text {in }}^{1}=\left(\Phi \left(k_{2}-\right.\right. & \left.\left.\frac{f}{\sqrt{\frac{1}{2 n}\left(1+\sqrt{1-\rho^{2}}\right)}}\right)+\Phi\left(k_{2}+\frac{f}{\sqrt{\frac{1}{2 n}\left(1+\sqrt{1-\rho^{2}}\right)}}\right)-1\right) \\
+ & 2\left\{\left(k_{1}+\frac{f}{\sqrt{\frac{1}{2 n}\left(1+\sqrt{1-\rho^{2}}\right)}}\right)\right. \\
& \left.-\left(k_{2}\right)\right\}\left\{\left(\Phi\left(k_{2}-\frac{f}{\sqrt{\frac{1}{2 n}\left(1+\sqrt{1-\rho^{2}}\right)}}\right)\right.\right. \\
& \left.\left.\left.\left.+\frac{f}{\sqrt{\frac{1}{2 n}\left(1+\sqrt{1-\rho^{2}}\right)}}\right)\right\}\left\{\begin{array}{l}
i \\
+
\end{array}\right)-1\right)\right\}
\end{aligned}
$$

The probability of repetition $P_{r e p}^{1}$ is given as

$$
\begin{aligned}
P_{\text {rep }}^{1}=2\left\{\left(k_{1}+\right.\right. & \left.\left.\frac{f}{\sqrt{\frac{1}{2 n}\left(1+\sqrt{1-\rho^{2}}\right)}}\right)-\left(k_{2}+\frac{f}{\sqrt{\frac{1}{2 n}\left(1+\sqrt{1-\rho^{2}}\right)}}\right)\right\}(1 \\
& \left.-\left[\Phi\left(k_{2}-\frac{f}{\sqrt{\frac{1}{2 n}\left(1+\sqrt{1-\rho^{2}}\right)}}\right)+\Phi\left(k_{2}+\frac{f}{\sqrt{\frac{1}{2 n}\left(1+\sqrt{1-\rho^{2}}\right)}}\right)-1\right]\right]^{i}
\end{aligned}
$$

Hence, the probability that the process is declared as in control for the shifted process is given as follows

$$
P_{\text {in }}^{1}=\frac{P_{\text {in, } 1}^{1}}{1-P_{\text {rep }}^{1}}=\frac{A_{1}^{1}+\left\{A_{2}^{1}+A_{3}^{1}\right\}\left\{A_{1}^{1}\right\}^{i}}{1-\left\{A_{2}^{1}+A_{3}^{1}\right\}\left(1-\left[A_{1}^{1}\right]^{i}\right)}
$$

So, the $A R L$ for the shifted process is given as follows

$$
A R L_{1}=\frac{1}{1-P_{\text {in }}^{1}}
$$

Using the above mentioned equations an R-language code program was written and run under the Monte Carlo simulation procedure. The ARLs for in-control and shifted processes were estimated for mean monitoring under the normal distribution for different process settings. The ARL analysis of the proposed scheme for different process settings with control chart coefficients has been given in Tables 1-6. Tables 1-3 are for the cases of $A R L_{0}=300$ and Tables 4-6 are for the cases of $A R L_{0}=370$. 
Table 1. The average run length $(A R L)$ analysis for 300 with $n=5$.

\begin{tabular}{|c|c|c|c|c|c|c|c|c|c|c|c|c|c|c|c|}
\hline \multirow{6}{*}{$f$} & \multicolumn{5}{|c|}{$\rho=0.3$} & \multicolumn{5}{|c|}{$\rho=0.6$} & \multicolumn{5}{|c|}{$\rho=0.9$} \\
\hline & $i=1$ & $i=2$ & $i=3$ & $i=4$ & $i=0$ & $i=1$ & $i=2$ & $i=3$ & $i=4$ & $i=0$ & $i=1$ & $i=2$ & $i=3$ & $i=4$ & $i=0$ \\
\hline & \multicolumn{4}{|c|}{$k_{1}$} & \multirow{2}{*}{$k$} & \multicolumn{4}{|c|}{$k_{1}$} & \multirow{2}{*}{$k$} & \multicolumn{4}{|c|}{$k_{1}$} & \multirow{2}{*}{$k$} \\
\hline & 2.9523 & 2.9701 & 2.9867 & 2.9953 & & 2.9607 & 2.9861 & 3.0027 & 3.0211 & & 2.9552 & 2.9706 & 3.0084 & 3.0170 & \\
\hline & \multicolumn{4}{|c|}{$k_{2}$} & \multirow{2}{*}{2.9352} & \multicolumn{4}{|c|}{$k_{2}$} & \multirow{2}{*}{2.9352} & \multicolumn{4}{|c|}{$k_{2}$} & \multirow{2}{*}{2.9352} \\
\hline & 1.1900 & 1.1537 & 1.1238 & 1.1316 & & 1.0728 & 1.0345 & 1.0323 & 1.0030 & & 1.1447 & 1.1493 & 1.0040 & 1.0214 & \\
\hline 0.0000 & 300.00 & 300.00 & 300.00 & 300.00 & 300.00 & 300.00 & 300.00 & 300.00 & 300.00 & 300.00 & 300.00 & 300.00 & 300.00 & 300.00 & 300.00 \\
\hline 0.0005 & 263.81 & 260.44 & 257.26 & 256.54 & 300.00 & 256.78 & 251.97 & 249.60 & 245.65 & 300.00 & 256.28 & 254.58 & 242.48 & 241.99 & 300.00 \\
\hline 0.0010 & 235.41 & 230.11 & 225.20 & 224.10 & 299.99 & 224.44 & 217.21 & 213.73 & 208.01 & 299.99 & 223.69 & 221.12 & 203.50 & 202.82 & 299.99 \\
\hline 0.0015 & 212.53 & 206.11 & 200.25 & 198.96 & 299.98 & 199.35 & 190.89 & 186.89 & 180.40 & 299.98 & 198.46 & 195.44 & 175.34 & 174.59 & 299.98 \\
\hline 0.0020 & 193.71 & 186.65 & 180.29 & 178.91 & 299.97 & 179.31 & 170.27 & 166.05 & 159.28 & 299.97 & 178.34 & 175.11 & 154.04 & 153.28 & 299.96 \\
\hline 0.0025 & 177.95 & 170.56 & 163.96 & 162.53 & 299.95 & 162.93 & 153.68 & 149.41 & 142.60 & 299.95 & 161.93 & 158.62 & 137.37 & 136.63 & 299.94 \\
\hline 0.0030 & 164.57 & 157.02 & 150.35 & 148.92 & 299.93 & 149.29 & 140.04 & 135.81 & 129.10 & 299.93 & 148.29 & 144.97 & 123.97 & 123.25 & 299.91 \\
\hline 0.0035 & 153.06 & 145.48 & 138.83 & 137.41 & 299.91 & 137.77 & 128.63 & 124.49 & 117.94 & 299.90 & 136.77 & 133.49 & 112.96 & 112.27 & 299.88 \\
\hline 0.0040 & 143.05 & 135.52 & 128.96 & 127.56 & 299.88 & 127.90 & 118.95 & 114.91 & 108.57 & 299.87 & 126.91 & 123.70 & 103.76 & 103.09 & 299.84 \\
\hline 0.0050 & 126.51 & 119.21 & 112.91 & 111.58 & 299.82 & 111.87 & 103.39 & 99.61 & 93.70 & 299.80 & 110.93 & 107.88 & 89.24 & 88.63 & 299.75 \\
\hline 0.0060 & 113.41 & 106.41 & 100.42 & 99.18 & 299.74 & 99.42 & 91.45 & 87.92 & 82.43 & 299.72 & 98.52 & 95.66 & 78.30 & 77.75 & 299.65 \\
\hline 0.0070 & 102.76 & 96.10 & 90.44 & 89.27 & 299.64 & 89.47 & 81.99 & 78.70 & 73.60 & 299.61 & 88.62 & 85.93 & 69.76 & 69.27 & 299.52 \\
\hline 0.0080 & 93.95 & 87.62 & 82.26 & 81.16 & 299.54 & 81.33 & 74.31 & 71.24 & 66.49 & 299.50 & 80.52 & 78.00 & 62.92 & 62.46 & 299.37 \\
\hline 0.0090 & 86.52 & 80.51 & 75.45 & 74.42 & 299.41 & 74.55 & 67.95 & 65.08 & 60.65 & 299.36 & 73.78 & 71.42 & 57.31 & 56.89 & 299.20 \\
\hline 0.0100 & 80.19 & 74.48 & 69.69 & 68.72 & 299.28 & 68.82 & 62.60 & 59.91 & 55.76 & 299.21 & 68.09 & 65.87 & 52.62 & 52.24 & 299.02 \\
\hline 0.0300 & 32.58 & 29.92 & 27.75 & 27.35 & 293.59 & 27.18 & 24.47 & 23.35 & 21.61 & 293.05 & 26.81 & 25.89 & 20.20 & 20.09 & 291.34 \\
\hline 0.0500 & 20.46 & 18.79 & 17.44 & 17.21 & 282.78 & 16.98 & 15.31 & 14.65 & 13.58 & 281.39 & 16.72 & 16.18 & 12.65 & 12.61 & 277.00 \\
\hline 0.1000 & 10.60 & 9.79 & 9.14 & 9.06 & 240.41 & 8.80 & 8.01 & 7.72 & 7.22 & 236.29 & 8.63 & 8.40 & 6.68 & 6.69 & 223.85 \\
\hline 0.2000 & 5.38 & 5.03 & 4.76 & 4.75 & 145.54 & 4.50 & 4.18 & 4.08 & 3.87 & 138.84 & 4.37 & 4.31 & 3.55 & 3.58 & 120.48 \\
\hline 0.3000 & 3.57 & 3.38 & 3.24 & 3.24 & 82.17 & 3.03 & 2.86 & 2.82 & 2.71 & 76.50 & 2.92 & 2.89 & 2.47 & 2.50 & 61.98 \\
\hline 0.4000 & 2.66 & 2.55 & 2.46 & 2.47 & 47.11 & 2.30 & 2.19 & 2.18 & 2.11 & 43.07 & 2.19 & 2.18 & 1.93 & 1.95 & 33.21 \\
\hline 0.5000 & 2.12 & 2.05 & 1.99 & 2.00 & 28.06 & 1.86 & 1.80 & 1.79 & 1.75 & 25.31 & 1.76 & 1.76 & 1.60 & 1.61 & 18.84 \\
\hline
\end{tabular}


Table 2. The $A R L$ analysis for 300 with $n=30$.

\begin{tabular}{|c|c|c|c|c|c|c|c|c|c|c|c|c|c|c|c|}
\hline \multirow{6}{*}{$f$} & \multicolumn{5}{|c|}{$\rho=0.3$} & \multicolumn{5}{|c|}{$\rho=0.6$} & \multicolumn{5}{|c|}{$\rho=0.9$} \\
\hline & $i=1$ & $i=2$ & $i=3$ & $i=4$ & $i=0$ & $i=1$ & $i=2$ & $i=3$ & $i=4$ & $i=0$ & $i=1$ & $i=2$ & $i=3$ & $i=4$ & $i=0$ \\
\hline & \multicolumn{4}{|c|}{$k_{1}$} & \multirow{2}{*}{$k$} & \multicolumn{4}{|c|}{$k_{1}$} & $k$ & \multicolumn{4}{|c|}{$k_{1}$} & \multirow{2}{*}{$k$} \\
\hline & 2.9623 & 2.9825 & 3.0048 & 3.0208 & & 2.9567 & 2.9757 & 3.0031 & 3.0115 & & 2.9596 & 2.9774 & 2.9905 & 3.0179 & \\
\hline & \multicolumn{4}{|c|}{$k_{2}$} & \multirow{2}{*}{2.9352} & \multicolumn{4}{|c|}{$k_{2}$} & 29352 & \multicolumn{4}{|c|}{$k_{2}$} & \multirow{2}{*}{2.9352} \\
\hline & 1.0556 & 1.0581 & 1.0223 & 1.0047 & & 1.1244 & 1.1075 & 1.0309 & 1.0475 & & 1.0874 & 1.0945 & 1.1003 & 1.0174 & \\
\hline 0.0000 & 300.00 & 300.00 & 300.00 & 300.00 & 300.00 & 300.00 & 300.00 & 300.00 & 300.00 & 300.00 & 300.00 & 300.00 & 300.00 & 300.00 & 300.00 \\
\hline 0.0010 & 213.49 & 209.53 & 202.35 & 197.59 & 299.99 & 216.78 & 211.79 & 200.61 & 199.93 & 299.99 & 206.55 & 203.28 & 200.87 & 188.58 & 299.99 \\
\hline 0.0015 & 165.73 & 161.03 & 152.74 & 147.40 & 299.96 & 169.72 & 163.71 & 150.76 & 150.01 & 299.95 & 157.52 & 153.77 & 151.05 & 137.61 & 299.94 \\
\hline 0.0020 & 135.45 & 130.79 & 122.71 & 117.60 & 299.90 & 139.46 & 133.45 & 120.80 & 120.09 & 299.89 & 127.31 & 123.68 & 121.07 & 108.40 & 299.87 \\
\hline 0.0025 & 114.53 & 110.13 & 102.58 & 97.87 & 299.83 & 118.36 & 112.65 & 100.81 & 100.16 & 299.81 & 106.83 & 103.46 & 101.05 & 89.46 & 299.76 \\
\hline 0.0030 & 99.22 & 95.13 & 88.15 & 83.84 & 299.73 & 102.82 & 97.47 & 86.52 & 85.94 & 299.71 & 92.04 & 88.94 & 86.74 & 76.19 & 299.63 \\
\hline 0.0035 & 87.53 & 83.73 & 77.29 & 73.34 & 299.61 & 90.88 & 85.91 & 75.79 & 75.27 & 299.58 & 80.85 & 78.01 & 75.99 & 66.37 & 299.47 \\
\hline 0.0040 & 78.30 & 74.78 & 68.83 & 65.20 & 299.47 & 81.44 & 76.80 & 67.45 & 66.97 & 299.42 & 72.09 & 69.47 & 67.62 & 58.81 & 299.28 \\
\hline 0.0045 & 70.84 & 67.57 & 62.06 & 58.70 & 299.30 & 73.77 & 69.45 & 60.77 & 60.34 & 299.25 & 65.05 & 62.63 & 60.93 & 52.81 & 299.05 \\
\hline 0.0050 & 59.51 & 56.66 & 51.87 & 48.98 & 298.91 & 62.09 & 58.31 & 50.75 & 50.38 & 298.82 & 54.42 & 52.34 & 50.88 & 43.89 & 298.53 \\
\hline 0.0060 & 51.31 & 48.80 & 44.58 & 42.04 & 298.44 & 53.61 & 50.26 & 43.59 & 43.28 & 298.31 & 46.79 & 44.97 & 43.69 & 37.57 & 297.88 \\
\hline 0.0070 & 45.10 & 42.87 & 39.10 & 36.85 & 297.88 & 47.17 & 44.17 & 38.22 & 37.95 & 297.70 & 41.04 & 39.43 & 38.30 & 32.87 & 297.12 \\
\hline 0.0080 & 40.24 & 38.23 & 34.83 & 32.81 & 297.23 & 42.11 & 39.41 & 34.04 & 33.80 & 297.00 & 36.55 & 35.11 & 34.10 & 29.23 & 296.25 \\
\hline 0.0090 & 36.32 & 34.50 & 31.42 & 29.59 & 296.51 & 38.04 & 35.58 & 30.69 & 30.48 & 296.21 & 32.95 & 31.65 & 30.75 & 26.33 & 295.27 \\
\hline 0.0100 & 33.11 & 31.44 & 28.62 & 26.95 & 295.70 & 34.69 & 32.43 & 27.95 & 27.77 & 295.34 & 30.00 & 28.82 & 28.00 & 23.96 & 294.18 \\
\hline 0.0300 & 12.01 & 11.48 & 10.51 & 9.94 & 264.92 & 12.60 & 11.83 & 10.24 & 10.22 & 262.26 & 10.80 & 10.45 & 10.21 & 8.81 & 254.02 \\
\hline 0.0500 & 7.37 & 7.10 & 6.56 & 6.25 & 218.00 & 7.71 & 7.29 & 6.39 & 6.40 & 212.83 & 6.60 & 6.44 & 6.33 & 5.54 & 197.59 \\
\hline 0.1000 & 3.77 & 3.69 & 3.48 & 3.36 & 112.69 & 3.91 & 3.76 & 3.38 & 3.41 & 106.24 & 3.36 & 3.32 & 3.30 & 2.98 & 89.15 \\
\hline 0.2000 & 1.94 & 1.93 & 1.87 & 1.84 & 29.51 & 1.97 & 1.94 & 1.82 & 1.84 & 26.65 & 1.73 & 1.73 & 1.73 & 1.64 & 19.89 \\
\hline 0.3000 & 1.37 & 1.37 & 1.35 & 1.34 & 9.85 & 1.37 & 1.36 & 1.32 & 1.33 & 8.74 & 1.24 & 1.25 & 1.25 & 1.22 & 6.26 \\
\hline 0.4000 & 1.14 & 1.14 & 1.13 & 1.13 & 4.23 & 1.13 & 1.13 & 1.11 & 1.12 & 3.76 & 1.07 & 1.07 & 1.07 & 1.06 & 2.75 \\
\hline 0.5000 & 1.05 & 1.05 & 1.04 & 1.04 & 2.30 & 1.04 & 1.04 & 1.03 & 1.03 & 2.08 & 1.02 & 1.02 & 1.02 & 1.01 & 1.62 \\
\hline
\end{tabular}


Table 3. The $A R L$ analysis for 300 with $n=60$.

\begin{tabular}{|c|c|c|c|c|c|c|c|c|c|c|c|c|c|c|c|}
\hline \multirow{6}{*}{$f$} & \multicolumn{5}{|c|}{$\rho=0.3$} & \multicolumn{5}{|c|}{$\rho=0.6$} & \multicolumn{5}{|c|}{$\rho=0.9$} \\
\hline & $i=1$ & $i=2$ & $i=3$ & $i=4$ & $i=0$ & $i=1$ & $i=2$ & $i=3$ & $i=4$ & $i=0$ & $i=1$ & $i=2$ & $i=3$ & $i=4$ & $i=0$ \\
\hline & \multicolumn{4}{|c|}{$k_{1}$} & \multirow{2}{*}{$k$} & \multicolumn{4}{|c|}{$k_{1}$} & \multirow{2}{*}{$k$} & \multicolumn{4}{|c|}{$k_{1}$} & \multirow{2}{*}{$k$} \\
\hline & 2.9590 & 2.9752 & 2.9953 & 3.0033 & & 2.9574 & 2.9849 & 3.0039 & 3.0150 & & 2.9618 & 2.9804 & 2.9915 & 2.9984 & \\
\hline & \multicolumn{4}{|c|}{$k_{2}$} & \multirow{2}{*}{2.9352} & \multicolumn{4}{|c|}{$k_{2}$} & \multirow{2}{*}{2.9352} & \multicolumn{4}{|c|}{$k_{2}$} & \multirow{2}{*}{2.9352} \\
\hline & 1.0940 & 1.1113 & 1.0727 & 1.0884 & & 1.1152 & 1.0424 & 1.0264 & 1.0307 & & 1.0612 & 1.0727 & 1.0948 & 1.1143 & \\
\hline 0.0000 & 300.00 & 300.00 & 300.00 & 300.00 & 300.00 & 300.00 & 300.00 & 300.00 & 300.00 & 300.00 & 300.00 & 300.00 & 300.00 & 300.00 & 300.00 \\
\hline 0.0005 & 194.36 & 192.08 & 184.45 & 183.81 & 299.98 & 193.57 & 181.61 & 175.88 & 173.54 & 299.98 & 180.38 & 176.89 & 176.05 & 175.99 & 299.97 \\
\hline 0.0010 & 143.77 & 141.32 & 133.25 & 132.60 & 299.91 & 142.90 & 130.29 & 124.51 & 122.20 & 299.91 & 128.99 & 125.49 & 124.67 & 124.62 & 299.88 \\
\hline 0.0015 & 114.09 & 111.81 & 104.35 & 103.77 & 299.80 & 113.28 & 101.63 & 96.43 & 94.38 & 299.79 & 100.41 & 97.28 & 96.56 & 96.53 & 299.73 \\
\hline 0.0020 & 94.58 & 92.52 & 85.79 & 85.28 & 299.65 & 93.83 & 83.33 & 78.72 & 76.92 & 299.62 & 82.21 & 79.45 & 78.83 & 78.82 & 299.53 \\
\hline 0.0025 & 80.78 & 78.92 & 72.86 & 72.41 & 299.46 & 80.09 & 70.64 & 66.54 & 64.95 & 299.41 & 69.61 & 67.16 & 66.63 & 66.63 & 299.26 \\
\hline 0.0030 & 70.50 & 68.82 & 63.33 & 62.94 & 299.22 & 69.87 & 61.31 & 57.64 & 56.23 & 299.15 & 60.36 & 58.18 & 57.71 & 57.72 & 298.94 \\
\hline 0.0035 & 62.54 & 61.02 & 56.02 & 55.67 & 298.94 & 61.96 & 54.17 & 50.86 & 49.59 & 298.85 & 53.29 & 51.33 & 50.92 & 50.93 & 298.55 \\
\hline 0.0040 & 56.20 & 54.82 & 50.24 & 49.92 & 298.61 & 55.67 & 48.53 & 45.51 & 44.37 & 298.49 & 47.71 & 45.93 & 45.57 & 45.59 & 298.11 \\
\hline 0.0050 & 46.74 & 45.57 & 41.66 & 41.41 & 297.84 & 46.28 & 40.19 & 37.64 & 36.68 & 297.65 & 39.45 & 37.96 & 37.67 & 37.70 & 297.06 \\
\hline 0.0060 & 40.01 & 39.00 & 35.61 & 35.40 & 296.89 & 39.60 & 34.31 & 32.11 & 31.29 & 296.63 & 33.63 & 32.37 & 32.13 & 32.17 & 295.78 \\
\hline 0.0070 & 34.98 & 34.10 & 31.11 & 30.93 & 295.78 & 34.61 & 29.94 & 28.02 & 27.30 & 295.43 & 29.32 & 28.22 & 28.02 & 28.07 & 294.29 \\
\hline 0.0080 & 31.07 & 30.30 & 27.63 & 27.48 & 294.51 & 30.74 & 26.57 & 24.86 & 24.23 & 294.05 & 25.99 & 25.03 & 24.86 & 24.91 & 292.58 \\
\hline 0.0090 & 27.96 & 27.27 & 24.86 & 24.73 & 293.09 & 27.66 & 23.89 & 22.36 & 21.80 & 292.51 & 23.35 & 22.49 & 22.35 & 22.40 & 290.66 \\
\hline 0.0100 & 25.41 & 24.80 & 22.60 & 22.49 & 291.51 & 25.13 & 21.71 & 20.32 & 19.82 & 290.80 & 21.19 & 20.42 & 20.30 & 20.36 & 288.55 \\
\hline 0.0300 & 9.05 & 8.91 & 8.20 & 8.21 & 236.55 & 8.93 & 7.81 & 7.39 & 7.26 & 232.24 & 7.50 & 7.31 & 7.33 & 7.39 & 219.25 \\
\hline 0.0500 & 5.52 & 5.47 & 5.09 & 5.12 & 168.68 & 5.44 & 4.83 & 4.62 & 4.57 & 162.16 & 4.58 & 4.50 & 4.54 & 4.60 & 143.87 \\
\hline 0.1000 & 2.80 & 2.81 & 2.68 & 2.70 & 63.22 & 2.75 & 2.52 & 2.46 & 2.46 & 58.32 & 2.34 & 2.34 & 2.37 & 2.41 & 46.09 \\
\hline 0.2000 & 1.47 & 1.48 & 1.45 & 1.46 & 11.67 & 1.43 & 1.38 & 1.37 & 1.37 & 10.37 & 1.28 & 1.29 & 1.30 & 1.31 & 7.45 \\
\hline 0.3000 & 1.12 & 1.12 & 1.11 & 1.11 & 3.58 & 1.10 & 1.09 & 1.08 & 1.08 & 3.19 & 1.05 & 1.05 & 1.05 & 1.05 & 2.36 \\
\hline 0.4000 & 1.02 & 1.02 & 1.02 & 1.02 & 1.73 & 1.02 & 1.01 & 1.01 & 1.01 & 1.59 & 1.00 & 1.00 & 1.01 & 1.01 & 1.31 \\
\hline 0.5000 & 1.00 & 1.00 & 1.00 & 1.00 & 1.19 & 1.00 & 1.00 & 1.00 & 1.00 & 1.14 & 1.00 & 1.00 & 1.00 & 1.00 & 1.05 \\
\hline
\end{tabular}


Table 4. The $A R L$ analysis for 370 with $n=5$.

\begin{tabular}{|c|c|c|c|c|c|c|c|c|c|c|c|c|c|c|c|}
\hline \multirow{6}{*}{$f$} & \multicolumn{5}{|c|}{$\rho=0.3$} & \multicolumn{5}{|c|}{$\rho=0.6$} & \multicolumn{5}{|c|}{$\rho=0.9$} \\
\hline & $i=1$ & $i=2$ & $i=3$ & $i=4$ & $i=0$ & $i=1$ & $i=2$ & $i=3$ & $i=4$ & $i=0$ & $i=1$ & $i=2$ & $i=3$ & $i=4$ & $i=0$ \\
\hline & \multicolumn{4}{|c|}{$k_{1}$} & \multirow{2}{*}{$k$} & \multicolumn{4}{|c|}{$k_{1}$} & $k$ & \multicolumn{4}{|c|}{$k_{1}$} & \multirow{2}{*}{$k$} \\
\hline & 3.0175 & 3.0326 & 3.0469 & 3.0759 & & 3.0104 & 3.0210 & 3.0335 & 3.0694 & & 3.0087 & 3.0278 & 3.0586 & 3.0706 & \\
\hline & \multicolumn{4}{|c|}{$k_{2}$} & \multirow{2}{*}{2.9997} & \multicolumn{4}{|c|}{$k_{2}$} & 2.9997 & \multicolumn{4}{|c|}{$k_{2}$} & \multirow{2}{*}{2.9997} \\
\hline & 1.1753 & 1.1665 & 1.1469 & 1.0418 & & 1.3191 & 1.2952 & 1.2528 & 1.0740 & & 1.3652 & 1.2146 & 1.0737 & 1.0678 & \\
\hline 0.0000 & 370.00 & 370.00 & 370.00 & 370.00 & 370.00 & 370.00 & 370.00 & 370.00 & 370.00 & 370.00 & 370.00 & 370.00 & 370.00 & 370.00 & 370.00 \\
\hline 0.0005 & 315.26 & 312.36 & 308.82 & 297.06 & 370.00 & 322.58 & 319.72 & 315.36 & 297.97 & 370.00 & 320.73 & 308.39 & 293.40 & 290.51 & 370.00 \\
\hline 0.0010 & 274.63 & 270.26 & 265.02 & 248.18 & 369.99 & 285.93 & 281.47 & 274.79 & 249.45 & 369.99 & 283.05 & 264.39 & 243.11 & 239.18 & 369.99 \\
\hline 0.0015 & 243.29 & 238.18 & 232.12 & 213.15 & 369.98 & 256.76 & 251.41 & 243.49 & 214.54 & 369.98 & 253.29 & 231.38 & 207.57 & 203.30 & 369.97 \\
\hline 0.0020 & 218.37 & 212.91 & 206.49 & 186.80 & 369.96 & 233.00 & 227.15 & 218.59 & 188.23 & 369.96 & 229.19 & 205.71 & 181.11 & 176.80 & 369.95 \\
\hline 0.0025 & 198.08 & 192.50 & 185.97 & 166.27 & 369.94 & 213.26 & 207.16 & 198.32 & 167.68 & 369.94 & 209.28 & 185.17 & 160.65 & 156.43 & 369.92 \\
\hline 0.0030 & 181.24 & 175.66 & 169.17 & 149.81 & 369.92 & 196.60 & 190.41 & 181.50 & 151.19 & 369.91 & 192.55 & 168.37 & 144.35 & 140.28 & 369.89 \\
\hline 0.0035 & 167.05 & 161.54 & 155.16 & 136.34 & 369.89 & 182.36 & 176.17 & 167.31 & 137.66 & 369.88 & 178.30 & 154.36 & 131.07 & 127.17 & 369.84 \\
\hline 0.0040 & 154.92 & 149.52 & 143.30 & 125.09 & 369.85 & 170.04 & 163.91 & 155.18 & 126.37 & 369.84 & 166.02 & 142.51 & 120.03 & 116.31 & 369.80 \\
\hline 0.0050 & 135.27 & 130.16 & 124.31 & 107.40 & 369.77 & 149.81 & 143.90 & 135.55 & 108.57 & 369.75 & 145.91 & 123.55 & 102.74 & 99.36 & 369.68 \\
\hline 0.0060 & 120.05 & 115.25 & 109.78 & 94.12 & 369.66 & 133.88 & 128.24 & 120.33 & 95.19 & 369.64 & 130.14 & 109.05 & 89.83 & 86.75 & 369.54 \\
\hline 0.0070 & 107.91 & 103.41 & 98.30 & 83.77 & 369.54 & 121.01 & 115.66 & 108.20 & 84.76 & 369.50 & 117.45 & 97.61 & 79.81 & 76.99 & 369.38 \\
\hline 0.0080 & 98.01 & 93.79 & 89.00 & 75.49 & 369.40 & 110.40 & 105.33 & 98.29 & 76.41 & 369.35 & 107.02 & 88.34 & 71.82 & 69.22 & 369.19 \\
\hline 0.0090 & 89.77 & 85.80 & 81.32 & 68.71 & 369.25 & 101.50 & 96.70 & 90.05 & 69.56 & 369.18 & 98.29 & 80.68 & 65.29 & 62.89 & 368.97 \\
\hline 0.0100 & 82.81 & 79.08 & 74.86 & 63.06 & 369.07 & 93.93 & 89.38 & 83.09 & 63.85 & 368.99 & 90.87 & 74.25 & 59.85 & 57.63 & 368.74 \\
\hline 0.0300 & 32.52 & 30.91 & 29.11 & 24.10 & 361.78 & 37.70 & 35.61 & 32.77 & 24.42 & 361.09 & 36.21 & 28.72 & 22.67 & 21.81 & 358.89 \\
\hline 0.0500 & 20.26 & 19.28 & 18.18 & 15.07 & 347.94 & 23.57 & 22.27 & 20.49 & 15.26 & 346.16 & 22.58 & 17.86 & 14.12 & 13.61 & 340.55 \\
\hline 0.1000 & 10.44 & 9.99 & 9.48 & 7.95 & 294.01 & 12.13 & 11.50 & 10.63 & 8.03 & 288.80 & 11.57 & 9.21 & 7.38 & 7.17 & 273.09 \\
\hline 0.2000 & 5.28 & 5.11 & 4.90 & 4.22 & 175.21 & 6.06 & 5.80 & 5.42 & 4.24 & 166.93 & 5.72 & 4.67 & 3.87 & 3.79 & 144.30 \\
\hline 0.3000 & 3.52 & 3.43 & 3.32 & 2.93 & 97.46 & 3.96 & 3.82 & 3.61 & 2.93 & 90.57 & 3.70 & 3.10 & 2.65 & 2.62 & 73.00 \\
\hline 0.4000 & 2.63 & 2.58 & 2.52 & 2.27 & 55.12 & 2.90 & 2.82 & 2.69 & 2.25 & 50.28 & 2.68 & 2.31 & 2.04 & 2.02 & 38.51 \\
\hline 0.5000 & 2.10 & 2.07 & 2.03 & 1.87 & 32.40 & 2.27 & 2.22 & 2.13 & 1.84 & 29.15 & 2.08 & 1.85 & 1.67 & 1.66 & 21.52 \\
\hline
\end{tabular}


Table 5. The $A R L$ analysis for 370 with $n=30$.

\begin{tabular}{|c|c|c|c|c|c|c|c|c|c|c|c|c|c|c|c|}
\hline \multirow{6}{*}{$f$} & \multicolumn{5}{|c|}{$\rho=0.3$} & \multicolumn{5}{|c|}{$\rho=0.6$} & \multicolumn{5}{|c|}{$\rho=0.9$} \\
\hline & $i=1$ & $i=2$ & $i=3$ & $i=4$ & $i=0$ & $i=1$ & $i=2$ & $i=3$ & $i=4$ & $i=0$ & $i=1$ & $i=2$ & $i=3$ & $i=4$ & $i=0$ \\
\hline & \multicolumn{4}{|c|}{$k_{1}$} & \multirow{2}{*}{$k$} & \multicolumn{4}{|c|}{$k_{1}$} & $k$ & \multicolumn{4}{|c|}{$k_{1}$} & \multirow{2}{*}{$k$} \\
\hline & 3.0131 & 3.0248 & 3.0473 & 3.0559 & & 3.0088 & 3.0276 & 3.0482 & 3.0714 & & 3.0124 & 3.0318 & 3.0509 & 3.0801 & \\
\hline & \multicolumn{4}{|c|}{$k_{2}$} & \multirow{2}{*}{2.9997} & \multicolumn{4}{|c|}{$k_{2}$} & 29997 & \multicolumn{4}{|c|}{$k_{2}$} & \multirow{2}{*}{2.9997} \\
\hline & 1.2566 & 1.2484 & 1.1445 & 1.1498 & & 1.3626 & 1.2171 & 1.1381 & 1.0641 & & 1.2719 & 1.1746 & 1.1203 & 1.0221 & \\
\hline 0.0000 & 370.00 & 370.00 & 370.00 & 370.00 & 370.00 & 370.00 & 370.00 & 370.00 & 370.00 & 370.00 & 370.00 & 370.00 & 370.00 & 370.00 & 370.00 \\
\hline 0.0005 & 268.36 & 264.68 & 248.82 & 246.99 & 369.99 & 276.66 & 257.79 & 244.58 & 230.95 & 369.98 & 258.36 & 243.33 & 232.51 & 214.40 & 369.98 \\
\hline 0.0010 & 210.53 & 206.06 & 187.50 & 185.44 & 369.94 & 220.93 & 197.83 & 182.73 & 167.96 & 369.94 & 198.49 & 181.32 & 169.59 & 151.05 & 369.92 \\
\hline 0.0015 & 173.22 & 168.72 & 150.46 & 148.49 & 369.87 & 183.89 & 160.53 & 145.89 & 132.04 & 369.86 & 161.15 & 144.52 & 133.52 & 116.68 & 369.83 \\
\hline 0.0020 & 147.14 & 142.85 & 125.67 & 123.86 & 369.78 & 157.49 & 135.07 & 121.44 & 108.81 & 369.76 & 135.64 & 120.16 & 110.14 & 95.10 & 369.70 \\
\hline 0.0025 & 127.89 & 123.86 & 107.92 & 106.26 & 369.65 & 137.72 & 116.60 & 104.03 & 92.57 & 369.62 & 117.10 & 102.84 & 93.75 & 80.29 & 369.52 \\
\hline 0.0030 & 113.10 & 109.34 & 94.57 & 93.06 & 369.50 & 122.36 & 102.57 & 91.00 & 80.56 & 369.45 & 103.03 & 89.89 & 81.62 & 69.49 & 369.32 \\
\hline 0.0035 & 101.38 & 97.87 & 84.18 & 82.79 & 369.32 & 110.08 & 91.57 & 80.89 & 71.33 & 369.26 & 91.97 & 79.85 & 72.28 & 61.28 & 369.07 \\
\hline 0.0040 & 91.86 & 88.58 & 75.85 & 74.57 & 369.11 & 100.04 & 82.70 & 72.81 & 64.02 & 369.03 & 83.06 & 71.83 & 64.88 & 54.82 & 368.79 \\
\hline 0.0050 & 77.34 & 74.46 & 63.34 & 62.24 & 368.61 & 84.61 & 69.30 & 60.70 & 53.15 & 368.49 & 69.59 & 59.83 & 53.86 & 45.30 & 368.11 \\
\hline 0.0060 & 66.78 & 64.24 & 54.39 & 53.44 & 368.00 & 73.30 & 59.64 & 52.06 & 45.46 & 367.83 & 59.87 & 51.28 & 46.06 & 38.63 & 367.28 \\
\hline 0.0070 & 58.77 & 56.48 & 47.67 & 46.83 & 367.28 & 64.66 & 52.35 & 45.60 & 39.74 & 367.05 & 52.54 & 44.88 & 40.25 & 33.69 & 366.30 \\
\hline 0.0080 & 52.47 & 50.41 & 42.44 & 41.69 & 366.45 & 57.84 & 46.66 & 40.57 & 35.31 & 366.15 & 46.81 & 39.90 & 35.76 & 29.89 & 365.19 \\
\hline 0.0090 & 47.39 & 45.52 & 38.26 & 37.58 & 365.52 & 52.32 & 42.09 & 36.55 & 31.78 & 365.14 & 42.21 & 35.92 & 32.17 & 26.87 & 363.92 \\
\hline 0.0100 & 43.21 & 41.49 & 34.83 & 34.21 & 364.48 & 47.76 & 38.33 & 33.26 & 28.90 & 364.02 & 38.43 & 32.67 & 29.25 & 24.42 & 362.53 \\
\hline 0.0300 & 15.63 & 15.05 & 12.63 & 12.45 & 325.15 & 17.37 & 13.85 & 12.04 & 10.52 & 321.76 & 13.76 & 11.71 & 10.54 & 8.89 & 311.28 \\
\hline 0.0500 & 9.52 & 9.20 & 7.80 & 7.72 & 265.71 & 10.55 & 8.47 & 7.43 & 6.57 & 259.21 & 8.34 & 7.17 & 6.51 & 5.58 & 240.06 \\
\hline 0.1000 & 4.75 & 4.63 & 4.03 & 4.01 & 134.74 & 5.19 & 4.28 & 3.84 & 3.48 & 126.83 & 4.12 & 3.64 & 3.38 & 3.00 & 105.95 \\
\hline 0.2000 & 2.30 & 2.27 & 2.07 & 2.07 & 34.12 & 2.42 & 2.12 & 1.98 & 1.86 & 30.73 & 1.98 & 1.83 & 1.76 & 1.64 & 22.76 \\
\hline 0.3000 & 1.52 & 1.51 & 1.43 & 1.44 & 11.04 & 1.55 & 1.43 & 1.38 & 1.33 & 9.76 & 1.34 & 1.29 & 1.26 & 1.22 & 6.92 \\
\hline 0.4000 & 1.20 & 1.20 & 1.16 & 1.17 & 4.61 & 1.21 & 1.16 & 1.14 & 1.12 & 4.08 & 1.10 & 1.09 & 1.08 & 1.06 & 2.95 \\
\hline 0.5000 & 1.07 & 1.07 & 1.05 & 1.06 & 2.44 & 1.07 & 1.05 & 1.04 & 1.04 & 2.20 & 1.03 & 1.02 & 1.02 & 1.01 & 1.69 \\
\hline
\end{tabular}


Table 6. The $A R L$ analysis for 370 with $n=60$.

\begin{tabular}{|c|c|c|c|c|c|c|c|c|c|c|c|c|c|c|c|}
\hline \multirow{6}{*}{$f$} & \multicolumn{5}{|c|}{$\rho=0.3$} & \multicolumn{5}{|c|}{$\rho=0.6$} & \multicolumn{5}{|c|}{$\rho=0.9$} \\
\hline & $i=1$ & $i=2$ & $i=3$ & $i=4$ & $i=0$ & $i=1$ & $i=2$ & $i=3$ & $i=4$ & $i=0$ & $i=1$ & $i=2$ & $i=3$ & $i=4$ & $i=0$ \\
\hline & \multicolumn{4}{|c|}{$k_{1}$} & \multirow{2}{*}{$k$} & \multicolumn{4}{|c|}{$k_{1}$} & \multirow{2}{*}{$k$} & \multicolumn{4}{|c|}{$k_{1}$} & \multirow{2}{*}{$k$} \\
\hline & 3.0161 & 3.0418 & 3.0597 & 3.0705 & & 3.0093 & 3.0220 & 3.0300 & 3.0548 & & 3.0218 & 3.0464 & 3.0652 & 3.0825 & \\
\hline & \multicolumn{4}{|c|}{$k_{2}$} & \multirow{2}{*}{2.9997} & \multicolumn{4}{|c|}{$k_{2}$} & \multirow{2}{*}{2.9997} & \multicolumn{4}{|c|}{$k_{2}$} & \multirow{2}{*}{2.9997} \\
\hline & 1.1988 & 1.0905 & 1.0675 & 1.0686 & & 1.3468 & 1.2827 & 1.2855 & 1.1562 & & 1.1118 & 1.0569 & 1.0372 & 1.0110 & \\
\hline 0.0000 & 370.00 & 370.00 & 370.00 & 370.00 & 370.00 & 370.00 & 370.00 & 370.00 & 370.00 & 370.00 & 370.00 & 370.00 & 370.00 & 370.00 & 370.00 \\
\hline 0.0005 & 233.92 & 215.25 & 207.54 & 204.36 & 369.97 & 248.56 & 237.87 & 235.91 & 214.44 & 369.97 & 209.48 & 196.44 & 188.70 & 180.84 & 369.96 \\
\hline 0.0010 & 171.04 & 151.85 & 144.32 & 141.30 & 369.89 & 187.14 & 175.31 & 173.21 & 151.07 & 369.88 & 146.13 & 133.80 & 126.78 & 119.83 & 369.85 \\
\hline 0.0015 & 134.82 & 117.34 & 110.69 & 108.05 & 369.75 & 150.06 & 138.82 & 136.87 & 116.68 & 369.73 & 112.22 & 101.50 & 95.53 & 89.69 & 369.66 \\
\hline 0.0020 & 111.27 & 95.64 & 89.81 & 87.52 & 369.55 & 125.25 & 114.92 & 113.16 & 95.08 & 369.51 & 91.10 & 81.80 & 76.68 & 71.73 & 369.39 \\
\hline 0.0025 & 94.72 & 80.73 & 75.59 & 73.58 & 369.30 & 107.48 & 98.05 & 96.47 & 80.26 & 369.24 & 76.68 & 68.52 & 64.08 & 59.80 & 369.05 \\
\hline 0.0030 & 82.47 & 69.86 & 65.27 & 63.49 & 369.00 & 94.12 & 85.51 & 84.08 & 69.46 & 368.91 & 66.20 & 58.97 & 55.06 & 51.30 & 368.63 \\
\hline 0.0035 & 73.02 & 61.58 & 57.45 & 55.86 & 368.63 & 83.72 & 75.82 & 74.51 & 61.23 & 368.52 & 58.25 & 51.77 & 48.28 & 44.94 & 368.14 \\
\hline 0.0040 & 65.52 & 55.06 & 51.32 & 49.88 & 368.22 & 75.39 & 68.10 & 66.91 & 54.76 & 368.07 & 52.01 & 46.14 & 43.00 & 40.00 & 367.58 \\
\hline 0.0050 & 54.36 & 45.46 & 42.31 & 41.11 & 367.22 & 62.88 & 56.59 & 55.59 & 45.23 & 366.99 & 42.84 & 37.92 & 35.31 & 32.82 & 366.23 \\
\hline 0.0060 & 46.45 & 38.72 & 36.02 & 34.99 & 366.01 & 53.92 & 48.42 & 47.55 & 38.55 & 365.67 & 36.42 & 32.21 & 29.98 & 27.86 & 364.59 \\
\hline 0.0070 & 40.55 & 33.74 & 31.37 & 30.48 & 364.59 & 47.20 & 42.32 & 41.56 & 33.61 & 364.13 & 31.69 & 28.00 & 26.07 & 24.22 & 362.67 \\
\hline 0.0080 & 35.99 & 29.90 & 27.80 & 27.02 & 362.96 & 41.97 & 37.58 & 36.91 & 29.80 & 362.37 & 28.04 & 24.78 & 23.07 & 21.44 & 360.48 \\
\hline 0.0090 & 32.35 & 26.85 & 24.97 & 24.27 & 361.13 & 37.78 & 33.80 & 33.20 & 26.78 & 360.39 & 25.15 & 22.23 & 20.70 & 19.25 & 358.03 \\
\hline 0.0100 & 29.38 & 24.38 & 22.67 & 22.04 & 359.11 & 34.35 & 30.72 & 30.18 & 24.32 & 358.20 & 22.81 & 20.16 & 18.78 & 17.47 & 355.32 \\
\hline 0.0300 & 10.37 & 8.68 & 8.15 & 7.99 & 289.14 & 12.13 & 10.89 & 10.74 & 8.75 & 283.68 & 7.99 & 7.17 & 6.77 & 6.38 & 267.29 \\
\hline 0.0500 & 6.28 & 5.34 & 5.06 & 4.99 & 203.94 & 7.28 & 6.59 & 6.53 & 5.41 & 195.84 & 4.85 & 4.42 & 4.22 & 4.02 & 173.16 \\
\hline 0.1000 & 3.12 & 2.75 & 2.66 & 2.65 & 74.50 & 3.50 & 3.24 & 3.23 & 2.79 & 68.59 & 2.45 & 2.30 & 2.25 & 2.19 & 53.89 \\
\hline 0.2000 & 1.55 & 1.46 & 1.44 & 1.44 & 13.15 & 1.63 & 1.57 & 1.57 & 1.46 & 11.65 & 1.31 & 1.28 & 1.27 & 1.26 & 8.28 \\
\hline 0.3000 & 1.14 & 1.12 & 1.11 & 1.11 & 3.87 & 1.16 & 1.14 & 1.14 & 1.11 & 3.44 & 1.05 & 1.05 & 1.05 & 1.04 & 2.51 \\
\hline 0.4000 & 1.03 & 1.02 & 1.02 & 1.02 & 1.81 & 1.03 & 1.02 & 1.02 & 1.02 & 1.65 & 1.01 & 1.00 & 1.00 & 1.00 & 1.34 \\
\hline 0.5000 & 1.00 & 1.00 & 1.00 & 1.00 & 1.22 & 1.00 & 1.00 & 1.00 & 1.00 & 1.16 & 1.00 & 1.00 & 1.00 & 1.00 & 1.06 \\
\hline
\end{tabular}


The following trends can be noted from Tables 1-6:

1. For all other fixed parameters, the values of $A R L$ decrease as the sample size increases. For example, when $A R L_{0}=300, i=1, \rho=0.3, f=0.0005$, the $A R L=263$ for $n=5$, and $A R L=213$ for $n=30$.

2. For all other fixed parameters, the values of $A R L$ decrease as $i$ increases from 1 to 4 .

3. It is found that the performance becomes better as this correlation gets stronger.

4. The performance in terms of $A R L$ becomes better as the subgroup size increases.

Figure 1 shows the ARLs according to the shift constant $f$ for different values of $n$ when $A R L_{0}=370$, $i=1$ and $\rho=0.6$. It shows that $A R L s$ decrease faster as the sample size increases. Figure 2 shows the $A R L s$ according to the shift constant $f$ for different values of $n$ when $A R L_{0}=370, i=3$, and $\rho=0.6$. It is seen that $A R L$ curves may meet each other as the sample size increases. It means that the difference between $A R L s$ decreases as the sample size increases for higher values of $i$.

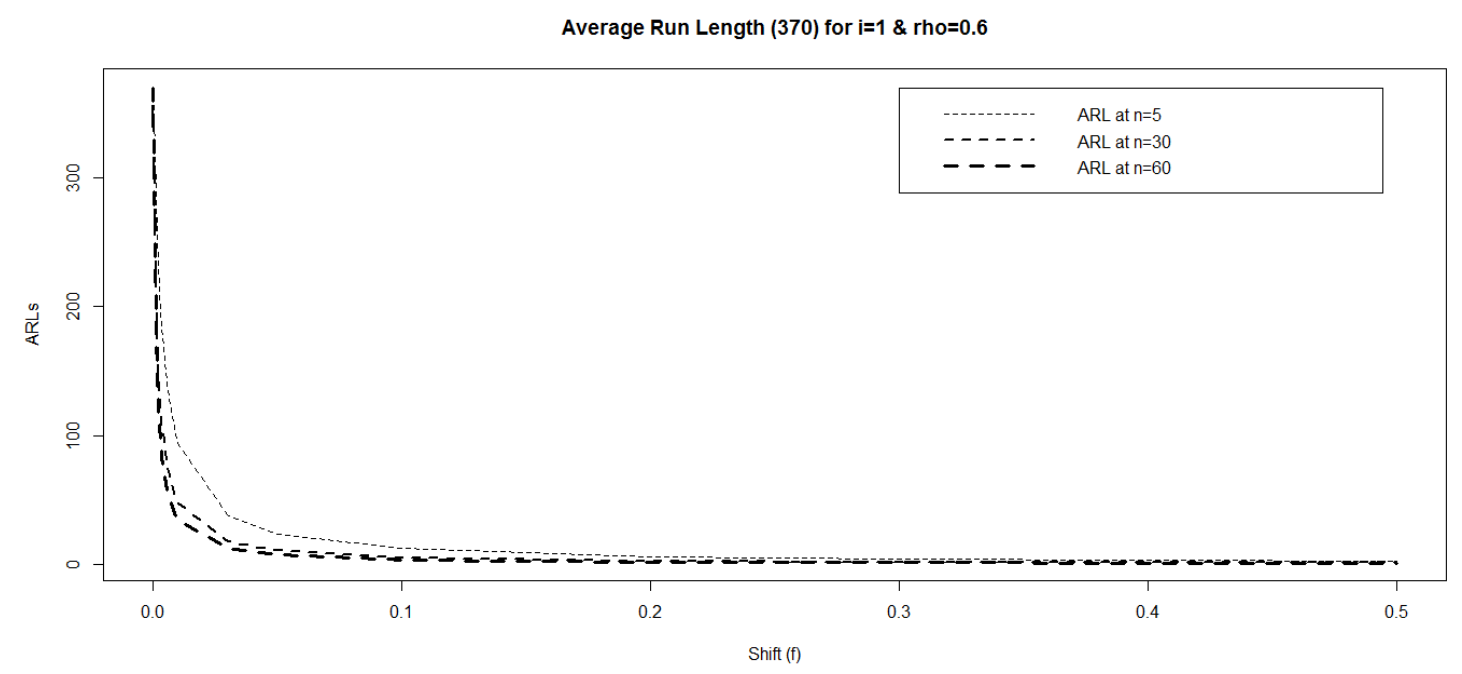

Figure 1. Trend of $A R L s$ according to shift constant when $A R L_{0}=370, i=1$ and $\rho=0.6$ with $n=5,30,60$.

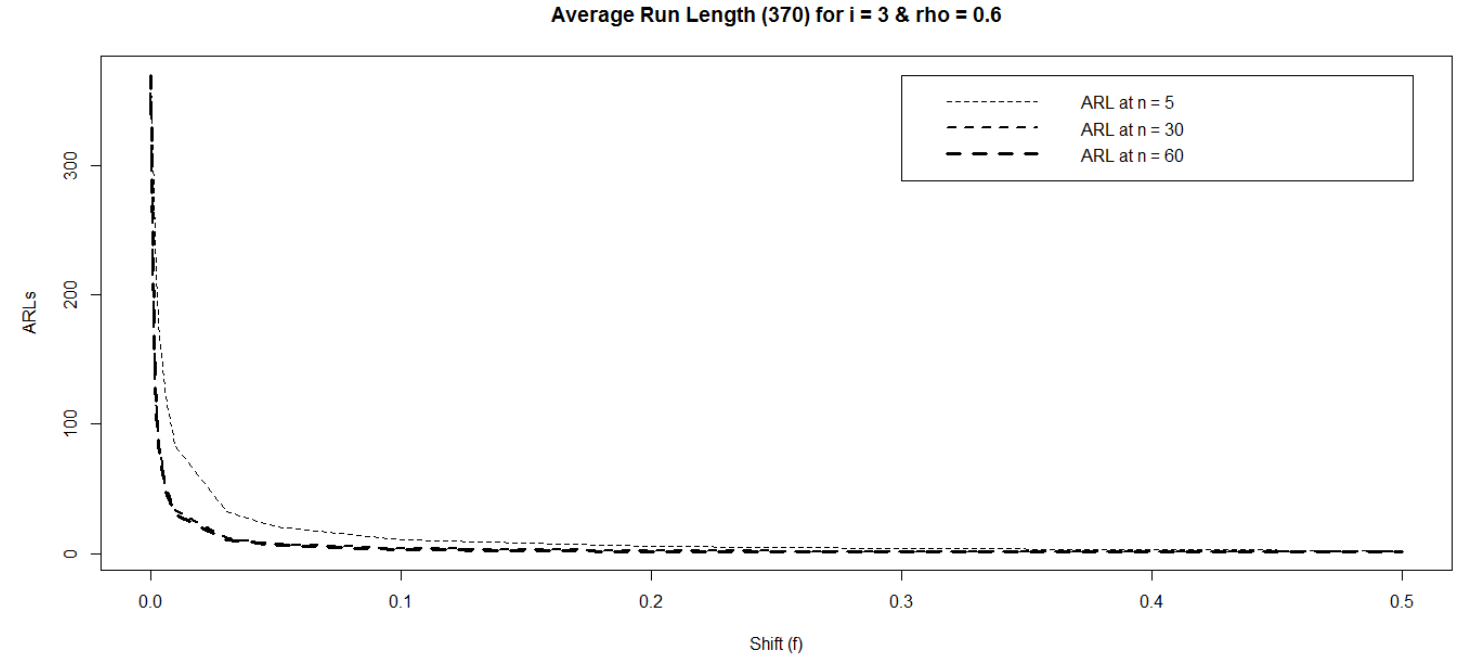

Figure 2. Trend of $A R L s$ according to shift constant when $A R L_{0}=370, i=3$ and $\rho=0.6$ with $n=5,30,60$. 


\section{Simulation Study}

In this section, we will compare the efficiency of the proposed control chart over the existing control charts using the repetitive sampling and multiple dependent state (MDS) sampling through the simulated data. The comparisons between charts will be given for the same values of control chart parameters.

The methodology of developing the proposed control chart using MDS repetitive sampling for process mean monitoring will be further explained via simulation data. In this simulation study, we consider the case of $A R L_{0}=370, n=30, i=2, k_{1}=3.0275, k_{2}=1.2171$, and $\rho=0.6$. A simulation data have been generated for constructing the control chart using the above mentioned parametric values. First 20 subgroups are generated from the in-control state of the process with mean zero and standard deviation 3 , while the next 18 subgroups are generated from an out-of-control process with $f=0.01$.

Figure 3 shows the proposed control chart, where an out-of-control signal appears at 38th subgroup. The same data is also plotted on an control chart using repetitive sampling in Figure 4 . From Figure 4, it is noted that all points lie between $L C L_{1}$ and $U C L_{1}$, which cannot detect a shift in the process. The Figure 5 shows the control chart based on the MDS sampling. The control statistics are also plotted on this chart, which again cannot detect a shift in the process. So, by comparing the proposed chart with existing charts, it can be seen that the proposed chart has ability to detect a shift in the process as compared to other charts.

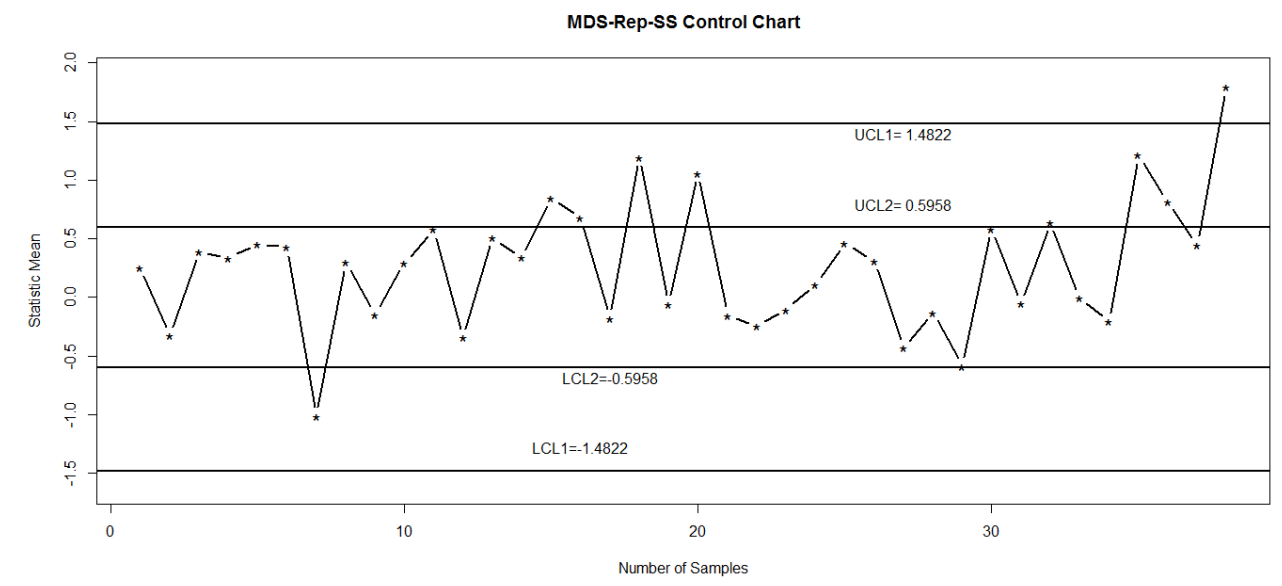

Figure 3. Proposed control chart for simulation data: $A R L_{0}=370$ for $i=2$ and $\rho=0.6$ with $n=30$.

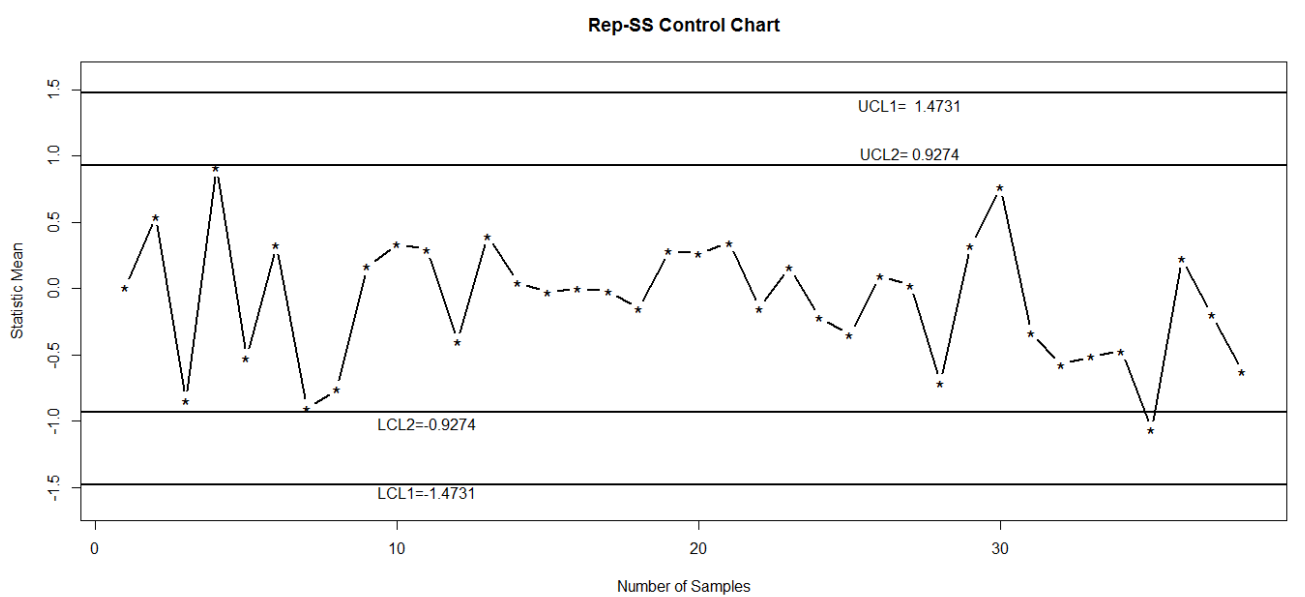

Figure 4. Control chart using repetitive sampling for simulation data: $A R L_{0}=370$ for $i=2$ and $\rho=0.6$ with $n=30$. 


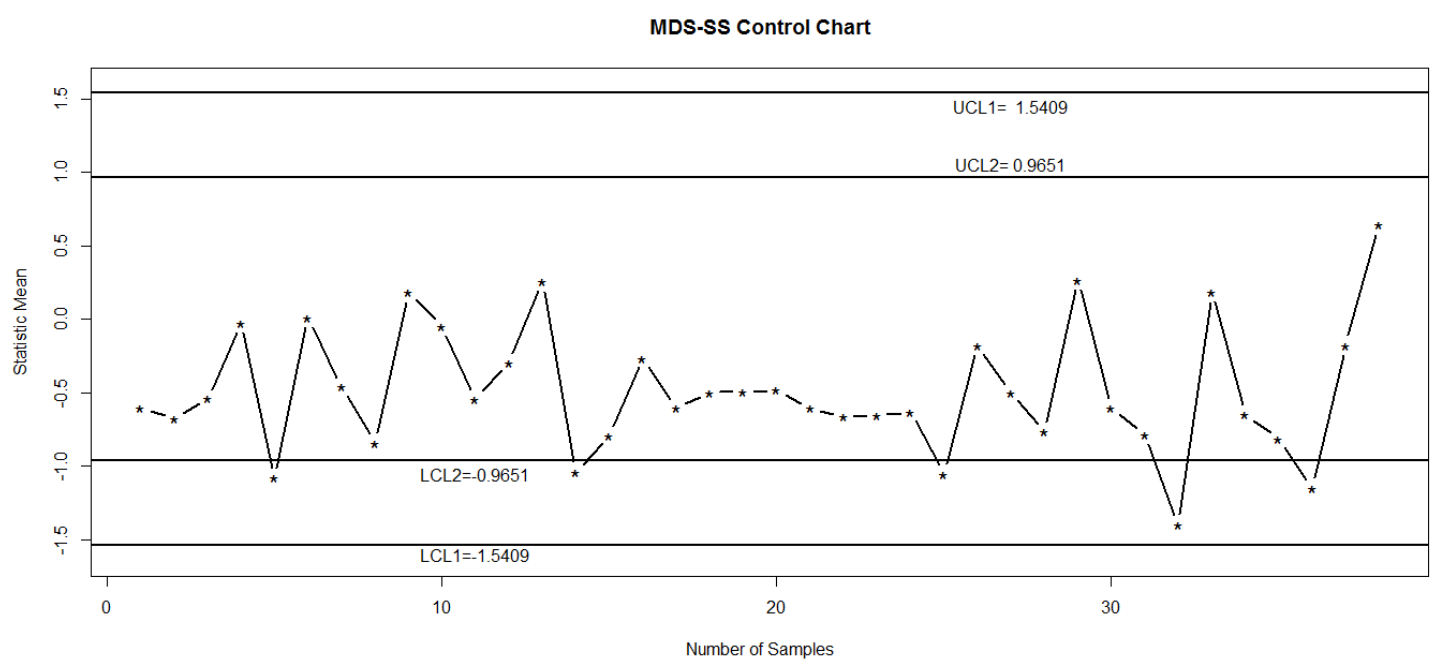

Figure 5. Control chart using MDS sampling for simulation data: $A R L_{0}=370$ for $i=2$ and $\rho=0.6$ with $n=30$.

\section{Comparison of Proposed Chart with Existing Charts}

In this section, we compare the performance of the proposed chart with some existing charts including Shewhart control chart, repetitive sampling control chart, and the multiple dependent state sampling control chart using successive sampling.

Tables 7-9 have been prepared for $\rho=0.3,0.6$ and 0.9 when $A R L_{0}=300, n=5$. The control chart coefficients were given. The $A R L$ values of the existing charts and the proposed chart for different shift levels $f=0.00,0.10,0.20,0.30,0.40$, and 0.50 have been estimated and given in Tables 7-9.

Table 7. ARL comparison when $A R L=300, n=5, r h o=0.3$.

\begin{tabular}{ccccc}
\hline \multirow{2}{*}{$f$} & Shewhart Control Chart & $\begin{array}{c}\text { Repetitive Sampling } \\
\text { Control Chart }\end{array}$ & MDS Sampling Chart & Proposed Chart \\
\cline { 3 - 5 } & \multirow{2}{*}{$\boldsymbol{k}=\mathbf{2 . 9 3 5 2}$} & $\boldsymbol{k}_{\mathbf{1}}=\mathbf{2 . 9 3 9 4}$ & $\boldsymbol{i}=\mathbf{2}$ & $\boldsymbol{i}=\mathbf{2}$ \\
\cline { 3 - 5 } & & $\boldsymbol{k}_{\mathbf{2}}=\mathbf{2 . 3 9 9 9}$ & $\boldsymbol{k}_{\mathbf{1}}=\mathbf{3 . 5 9 8 7}$ & $\boldsymbol{k}_{\mathbf{1}}=\mathbf{2 . 9 7 0 1}$ \\
\hline 0.0 & 300.08 & 300 & 30004 & $\boldsymbol{k}_{\mathbf{2}}=\mathbf{1 . 1 5 3 7}$ \\
0.1 & 241.6 & 239.91 & 234.75 & 300 \\
0.2 & 147.47 & 144.2 & 129.56 & 9.79 \\
0.3 & 83.83 & 80.46 & 63.2 & 5.03 \\
0.4 & 48.32 & 45.36 & 31.04 & 3.38 \\
0.5 & 28.89 & 26.42 & 16.19 & 2.55 \\
\hline
\end{tabular}

Table 8. ARL comparison when $A R L=300, n=5, r h o=0.6$.

\begin{tabular}{ccccc}
\hline \multirow{2}{*}{$f$} & Shewhart Control Chart & $\begin{array}{c}\text { Repetitive Sampling } \\
\text { Control Chart }\end{array}$ & MDS Sampling Chart & Proposed Chart \\
\cline { 3 - 5 } & \multirow{2}{*}{$\boldsymbol{k}=\mathbf{2 . 9 3 5 2}$} & $\boldsymbol{k}_{\mathbf{1}}=\mathbf{2 . 9 3 9 4}$ & $\boldsymbol{i}=\mathbf{2}$ & $\boldsymbol{i}=\mathbf{2}$ \\
\cline { 3 - 5 } & & $\boldsymbol{k}_{\mathbf{2}}=\mathbf{2 . 3 9 9 9}$ & $\boldsymbol{k}_{\mathbf{1}}=\mathbf{3 . 5 8 8 1}$ & $\boldsymbol{k}_{\mathbf{1}}=\mathbf{2 . 9 6 6 1 2}$ \\
\hline 0 & 300.08 & 300 & 300 & $\boldsymbol{k}_{\mathbf{2}}=\mathbf{1 . 0 3 4 5}$ \\
\hline 0.1 & 241.6 & 235.76 & 230.13 & 300 \\
0.2 & 147.47 & 137.45 & 122.21 & 8.01 \\
0.3 & 83.83 & 74.77 & 57.66 & 4.18 \\
0.4 & 48.32 & 41.33 & 27.69 & 2.86 \\
0.5 & 28.89 & 23.71 & 14.26 & 1.8 \\
\hline
\end{tabular}


Table 9. $A R L$ comparison when $A R L=300, n=5, r h o=0.9$.

\begin{tabular}{ccccc}
\hline \multirow{2}{*}{$f$} & Shewhart Control Chart & $\begin{array}{c}\text { Repetitive Sampling } \\
\text { Control Chart }\end{array}$ & MDS Sampling Chart & Proposed Chart \\
\cline { 3 - 5 } & \multirow{2}{*}{$\boldsymbol{k}=\mathbf{2 . 9 3 5 2}$} & $\boldsymbol{k}_{\mathbf{1}}=\mathbf{2 . 9 3 9 4}$ & $\boldsymbol{i}=\mathbf{2}$ & $\boldsymbol{i}=\mathbf{2}$ \\
\cline { 3 - 5 } & & $\boldsymbol{k}_{\mathbf{2}}=\mathbf{2 . 3 9 9 9}$ & $\boldsymbol{k}_{\mathbf{1}}=\mathbf{2 . 9 5 7 9}$ & $\boldsymbol{k}_{\mathbf{1}}=\mathbf{2 . 9 7 0 6}$ \\
\hline 0 & 300.08 & 300 & 300 & $\boldsymbol{k}_{\mathbf{2}}=\mathbf{1 . 1 4 9 3}$ \\
\hline 0.1 & 241.6 & 223.2 & 220.97 & 300 \\
0.2 & 147.47 & 118.96 & 114.85 & 8.4 \\
0.3 & 83.83 & 60.23 & 56.16 & 4.31 \\
0.4 & 48.32 & 31.52 & 28.32 & 2.89 \\
0.5 & 28.89 & 17.34 & 15.1 & 2.18 \\
\hline
\end{tabular}

It can be found from these tables that the proposed chart is much faster in detecting an out-of-control process. For instance, if a process faces a shift of 0.30 then the Shewhart chart will indicate the out-of-control process after an average of 83.83 samples, Shewhart control chart 82.19, repetitive sampling control chart 80.46 and multiple dependent state sampling chart will indicate an out-of-control process in 63.20 samples while the proposed successive sampling scheme detects the same process in only 3.38 average samples. The similar detecting ability of the proposed chart for $\rho=0.6$ and 0.9 can be observed in Tables 7-9.

\section{Concluding Remarks}

In this article, we presented a control chart for process mean monitoring using successive sampling over two occasions and MDS sampling. The coefficients of the proposed control charts have been estimated for the target in-control ARL. Extensive tables have been constructed for different process settings to evaluate the monitoring ability of the proposed scheme. It has been observed that the proposed chart is comparatively efficient than four other existing charts in terms of the out-of-control ARLs. The proposed chart using successive sampling works well when the subgroup size is large. The performance in terms of $A R L$ becomes better as the subgroup size increases. It is found that the performance becomes better as this correlation gets stronger. The proposed chart will be an efficient addition in the toolkit of the quality control personnel. The proposed control chart can be only used when the quality of interest follows the normal distribution. The proposed control chart for multivariate distribution can be considered as future research.

Author Contributions: Conceptualization, M.S.A., M.A., C.-H.J., and K.K.; Methodology, M.S.A., M.A., C.-H.J., and K.K.; Software, M.S.A., M.A., C.-H.J., and K.K.; Validation, M.S.A., M.A., C.-H.J. and K.K.; Formal Analysis, M.S.A., M.A., C.-H.J., and K.K.; Writing-Original Draft Preparation, M.A. and C.-H.J.; Writing-Review and Editing, M.S.A., M.A., C.-H.J., and K.K.

Funding: This research received no external funding.

Acknowledgments: The authors are deeply thankful to the editor and the reviewers for their valuable suggestions to improve the quality of this manuscript. This article was funded by the Deanship of Scientific Research (DSR) at King Abdulaziz University, Jeddah. The author, Muhammad Aslam, therefore, acknowledge with thanks DSR technical and financial support.

Conflicts of Interest: The authors declare no conflict of interest.

\section{References}

1. He, D.; Grigoryan, A.; Sigh, M. Design of double-and triple-sampling X-bar control charts using genetic algorithms. Int. J. Prod. Res. 2002, 40, 1387-1404. [CrossRef]

2. Montgomery, D.C. Introduction to Statistical Quality Control, 6th ed.; John Wiley \& Sons, Inc.: New York, NY, USA, 2009.

3. Chandara, M.J. Statistical Quality Control; CRC Press LLC: Boca Raton, FL, USA, 2001. 
4. Singh, H.P.; Vishwakarma, G.K. A general procedure for estimating population mean in successive sampling. Commun. Stat. Theory Methods 2009, 38, 293-308. [CrossRef]

5. Rueda, M.; Martinez, S.; Arcos, A.; Munoz, J.F. Mean estimation under successive sampling with calibration estimators. Commun. Stat. Theory Methods 2009, 38, 808-827. [CrossRef]

6. Valliant, R. Finite Population Sampling and Inference A Prediction Approach; John Wiley: New York, NY, USA, 2000.

7. Singh, H.P.; Tailor, R.; Singh, S.; Kim, J.M. Quantile estimation in successive sampling. J. Korean Stat. Soc. 2007, 36, 543-556.

8. Rueda, M.D.M.; Muñoz, J.F.; Arcos, A. Successive sampling to estimate quantiles with P-Auxiliary Variables. Qual. Quant. 2008, 42, 427-443. [CrossRef]

9. Singh, G.; Priyanka, K. Estimation of population mean at current occasion in successive sampling under a super-population model. Model Assist. Stat. Appl. 2007, 2, 189-200.

10. Cochran, W.G. Sampling Techniques; John Wiley \& Sons: New York, NY, USA, 2007.

11. Azam, M.; Nawaz, S.; Arshad, A.; Aslam, M. Acceptance Sampling Plan Using Successive Sampling over Two Successive Occasions. J. Test. Eval. 2016, 44, 2024-2032. [CrossRef]

12. Jessen, R.J. Statistical investigation of a sample survey for obtaining farm facts. Res. Bull. (Iowa Agric. Home Econ. Exp. Stn.) 1942, 26, 1 .

13. Azam, M.; Arshad, A.; Aslam, M.; Jun, C.-H. A Control Chart for Monitoring the Process Mean Using Successive Sampling over Two Occasions. Arab. J. Sci. Eng. 2017, 42, 2915-2926. [CrossRef]

14. Sen, A. Successive sampling with two auxiliary variables. Sankhyā Indian J. Stat. Ser. B 1971, 33, 371-378.

15. Li, Z.H.; Zou, C.L.; Gong, Z.; Wang, Z.J. The computation of average run length and average time to signal: An overview. J. Stat. Comput. Simul. 2014, 84, 1779-1802. [CrossRef]

16. Phanyaem, S.; Areepong, Y.; Sukparungsee, S. Numerical Integration of Average Run Length of CUSUM Control Chart for ARMA Process. Int. J. Appl. Phys. Math. 2014, 4, 232-235. [CrossRef]

17. Knoth, S. Accurate ARL calculation for EWMA control charts monitoring normal mean and variance simultaneously. Sequential Anal. 2007, 26, 251-263. [CrossRef]

18. Aslam, M.; Rao, G.S.; Ahmad, L.; Jun, C.-H. A control chart for multivariate Poisson distribution using repetitive sampling. J. Appl. Stat. 2017, 44, 123-136. [CrossRef]

19. Aslam, M.; Saghir, A.; Ahamd, L.; Jun, C.-H.; Hussain, J. A control chart for COM-Poisson distribution using a modified EWMA statistic. J. Stat. Comput. Simul. 2017, 87, 1-12. [CrossRef]

20. Ahmad, L.; Rafiq, I.; Aldosari, M.S.; Aslam, M. Mixed Repetitive Sampling Plan Using EWMA. Adv. Appl. Stat. 2017, 51, 167-186. [CrossRef]

21. Artes Rodriguez, E.M.; Garcia Luengo, A.V. Estimation of current population ratio in successive sampling. J. Ind. Soc. Agric. Stat. 2001, 54, 342-354.

22. Rueda, M.; Muñoz, J.F.; González, S.; Arcos, A. Estimating quantiles under sampling on two occasions with arbitrary sample designs. Comput. Stat. Data Anal. 2007, 51, 6596-6613. [CrossRef]

23. Kowalczyk, B. Estimation of Population Parameters Using Information from Previous Period in the Case of Overlapping Samples-Simulation Study. Metody Ilościowe w Badaniach Ekonomicznych 2013, 14, $283-292$.

24. Aslam, M.; Nazir, A.; Jun, C.-H. A new attribute control chart using multiple dependent state sampling. Trans. Inst. Meas. Control 2015, 37, 569-576. [CrossRef]

25. Chiu, J.-E.; Kuo, T.-I. Attribute control chart for multivariate Poisson distribution. Commun. Stat. Theory Methods 2007, 37, 146-158. [CrossRef]

(C) 2018 by the authors. Licensee MDPI, Basel, Switzerland. This article is an open access article distributed under the terms and conditions of the Creative Commons Attribution (CC BY) license (http://creativecommons.org/licenses/by/4.0/). 